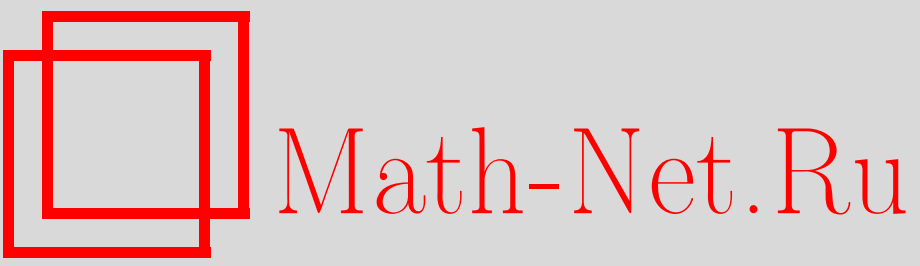

Ю. Г. Решетняк, Теория кривых в дифференциальной геометрии с точки зрения теории функций действительной переменной, УМH, 2005, том 60, выпуск 6, 157174

DOI: https://doi.org/10.4213/rm1681

Использование Общероссийского математического портала Math-Net.Ru подразумевает, что вы прочитали и согласны с пользовательским соглашением http://www . mathnet.ru/rus/agreement

Параметры загрузки:

IP: 34.229 .45 .116

26 апреля 2023 г., $14: 31: 00$ 


\section{ТЕОРИЯ КРИВЫХ В ДИФФЕРЕНЦИАЛЬНОЙ ГЕОМЕТРИИ С ТОЧКИ ЗРЕНИЯ ТЕОРИИ ФУНКЦИЙ ДЕЙСТВИТЕЛЬНОЙ ПЕРЕМЕННОЙ}

\section{Ю. Г. РЕШЕТнЯК}

Статья содержит краткий обзор теории кривых, построенной А. Д. Александровым и автором. Определяются основные понятия теории. Приводятся формулировки некоторых важнейших ее резултатов и описьваются применяемые методы исследования. Главными являются: метод, основанньй на использовании понятий индикатрисы касательных кривой в $\mathbb{R}^{n}$ и развертки сферической кривой, и метод, опирающийся на интегрально-геометрические соотношения типа тех, которые были найдены И. Фари и Дж. Милнором. Интегрально-геометрический метод для решения задач теории кривых предложен и реализован автором.

Библиография: 30 названий

\section{СОДЕРЖАНИЕ}

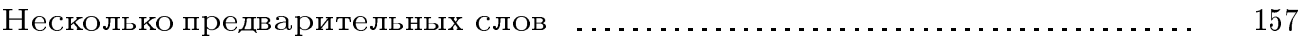

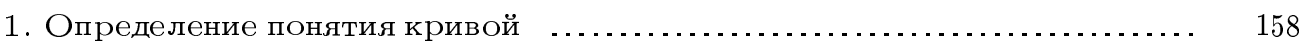

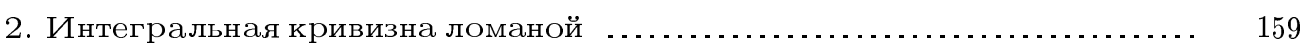

3. Поворот кривой, свойства кривых конечного поворота . . . . . . . . . . . . 161

4. Основное интегрально-геометрическое соотношение для поворота

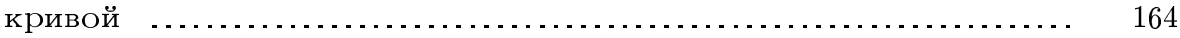

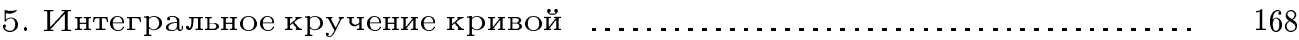

6. Восстановление кривой по интегральной кривизне и интегральному кручению ................................................... 171

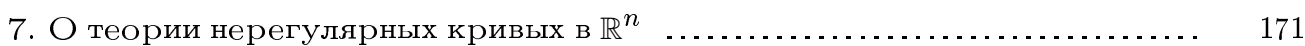

Список литературы .................................................. 173

Я благодарю Организационный комитет за предоставленную мне высокую честь участвовать в конференции, посвященной 100-летнему юбилею выдаюшегося математика и замечательного человека, академика Сергея Михайловича Никольского.

\section{Несколько предварительных слов}

Цель моего сообшения - рассказать об одной теории, в разработке которой я принимал участие. Теория эта довольно старая. В исследованиях Александра Даниловича Александрова по теории выпуклых поверхностей весьма эффективньм оказался 
предложенньй им метод, при котором тот или иной факт устанавливался сначала для многогранных поверхностей, а затем, с помошью предельного перехода, доказьвалась его справедливость для произвольных вьпуклых поверхностей. Именно на этом пути им было найдено чисто геометрическое решение знаменитой проблемы Вейля о реализации метрики положительной кривизны, заданной на сфере, как внутренней метрики замкнутой вьпуклой поверхности. В связи с этим Александр Данилович говорил, что надо действовать согласно принципу “назад $к$ Евклиду!”. Как иллюстрацию этой своей идеи А. Д. Александров предлагал определять основные понятия теории кривых сначала для объектов, в геометрическом отношении наиболее простых, а именно для ломаных, т.е. для кривых, составленных из последовательно примыкаюших друг к другу прямолинейных отрезков. Для произвольных кривых предлагалось определять соответствуюшие характеристики путем предельного перехода. (О каких именно характеристиках идет речь, будет сказано чуть позже.) О таком способе построения теории кривых в дифференциальной геометрии Александр Данилович рассказал в 1946 г. в своих выступлениях на научной сессии математико-механического факультета Ленинградского университета, тезисы которого были опубликованы [1], и затем в 1947 г. на заседании Московского математического общества (тезисы выступления опубликованы в том же году в "Успехах математических наук" [2]).

Теория кривых была темой кандидатской диссертации А.Я. Юсупова 1949 г. [3]. В 1949 г. на геометрическом семинаре, руководимом Александром Даниловичем Александровым, в Ленинградском университете А. Я. Юсупов рассказьвал доказательство той части теоремы 1 А. Д. Александрова (см. ниже), где речь идет об индикатрисе касательных. Тогда я предложил другое доказательство этого утверждения, которое понравилось Александру Даниловичу. Он сказал, обращаясь ко мне: “Вы будете писать обзорную статью о теории кривых для 'Успехов математических наук' !' И таким образом, я был вовлечен в работу над теорией кривых. В процессе реализации программы А. Д. Александрова первоначальньй замысел претерпел некоторые изменения. Результаты работы над теорией кривых по А. Д. Александрову представлены в книге "General theory of irregular curves" ( "Общая теория нерегулярных кривых") [4], опубликованной мною в соавторстве с А. Д. Александровым на английском языке сорок лет спустя в 1989 г. (первый ее вариант я написал еше в 1953 г.). На русском языке по этой теме опубликованы работы [1]-[3], [5]-[8] (среди которых кандидатская диссертация Ю.Г. Решетняка [6], зашишенная в 1954 г.), а также работы [9]-[25] и некоторые другие.

В 2001 г. в работе [22] мною было показано, как строить теорию кривых по А. Д. Александрову в $\mathbb{R}^{n}$ для произвольного $n$.

\section{1. Определение понятия кривой}

Термину “кривая" в разных разделах математики приписьвается разный смысл, и свой доклад я начну с определения того, что́ понимается под кривой в теории, о которой здесь говорится, - речь идет о кривых в смысле определения, данного М. Фреше. Далее $\mathscr{M}$ означает произвольное хаусдорфово топологическое пространство.

Кривая в смысле $\Phi$ реше в топологическом пространстве $\mathscr{M}$ есть компактное множество $E \subset \mathscr{M}$ с заданным порядком обхода его точек. Порядок обхода определяется указанием непрерьвного отображения $x$ отрезка $[a, b]$ числовой прямой $\mathbb{R}$ на множес- 
тво $E$. Всякое такое отображение назьвается параметризацией данной кривой. Необходимо указать, при каких условиях мы считаем, что непрерьвные отображения $x:[a, b] \rightarrow \mathscr{M}$ и $y:[c, d] \rightarrow \mathscr{M}$ определяют один и тот же порядок обхода точек некоторого множества, т.е. являются параметризациями одной и той же кривой.

Я опускаю точные определения, ввиду их громоздкости. Определение того, что́ есть кривая в смысле М. Фреше, может быть найдено во многих источниках, например, в указанной вьше книге [4] или в статье [22].

Так как кривая однозначно определяется указанием какой-либо своей параметризации, то я далее буду говорить "кривая $x:[a, b] \rightarrow \mathscr{M}$ ", каждый раз имея в виду кривую, одна из параметризаций которой есть данная функция $x$.

В теории кривых рассматриваются также и замкнутые кривые. Замкнутая кривая в смысле Фреше также есть множество с заданным порядком его обхода. При этом должно вьполняться условие - обход заканчивается в той же точке, в которой он начинается. Выбор начальной точки обхода при этом несуществен. Формально замкнутая кривая определяется заданием пути $x:[a, b] \rightarrow \mathscr{M}$ такого, что $x(a)=x(b)$. Всякий такой путь называется параметризацией замкнутой кривой.

Понятие длины кривой в метрическом пространстве здесь предполагается известным. Если $K-$ кривая, то $s(K)$ далее означает ее длину.

\section{2. Интегральная кривизна ломаной}

Ломаной в пространстве $\mathbb{R}^{n}$ называется объект, получаемьй следующим образом. Задается некоторая конечная последовательность $X_{0}, X_{1}, \ldots, X_{m}$ точек пространства $\mathbb{R}^{n}$ и затем точки $X_{0}$ и $X_{1}, X_{1}$ и $X_{2}, \ldots$, и, наконец, точки $X_{m-1}$ и $X_{m}$ соединяются прямолинейными отрезками. Точки $X_{i}, i=0,1, \ldots, m$, назьваются вершинами данной ломаной, векторы $\mathbf{a}_{i}=\overrightarrow{X_{i-1} X_{i}}$ назьваются ее звеньями.

Ломаную можно рассматривать как кривую, если мы последовательно параметризуем отрезки $X_{i-1} X_{i}$ так, чтобы областями изменения параметра были последовательно примькающие друг к другу отрезки. В результате получим непрерьвную функцию $x:[a, b] \rightarrow \mathbb{R}^{n}$, которая и будет параметризацией этой кривой.

Пусть $K$ есть кривая в пространстве $\mathbb{R}^{n}$, а $x:[a, b] \rightarrow \mathbb{R}^{n}$ есть произвольная ее параметризация. Зададим последовательность $\xi=\left\{t_{0} \leqslant t_{1} \leqslant \cdots \leqslant t_{m}\right\}$ и положим $X_{i}=x\left(t_{i}\right)$. Мы будем говорить, что последовательность точек $X_{i}$ определяет некоторую цепочку точек кривой $K$. Соединяя последовательно точки $X_{0}$ и $X_{1}, X_{1}$ и $X_{2}$ и т. д. прямолинейньми отрезками, мы получим некоторую ломаную $L_{\xi}$. Всякую ломаную, получаемую таким построением, естественно называть ломаной, вписанной в данную кривую $K$. Положим

$$
\delta(\xi)=\max \left\{t_{0}-a, t_{1}-t_{0}, \ldots, t_{m}-t_{m-1}, b-t_{m}\right\}
$$

Основные характеристики, относящиеся к ломаным, есть интегральная кривизна и интегральное кручение ломаной. Интегральную кривизну будем также назьвать поворотом ломаной. Для произвольной кривой поворот и интегральное кручение определяются как пределы соответствуюших характеристик вписанных ломаных.

Пусть $L$ есть ломаная в пространстве $\mathbb{R}^{n}, X_{0}, X_{1}, \ldots, X_{m}$ - ее последовательные вершины. Пусть $0<i<m$. Точка $X_{i}$ является конщом вектора $\mathbf{a}_{i}=\overrightarrow{X_{i-1} X_{i}}$ и 
началом вектора $\mathbf{a}_{i+1}=\overrightarrow{X_{i} X_{i+1}}$. Полагаем $\theta_{i}=\angle\left(\mathbf{a}_{i}, \mathbf{a}_{i+1}\right)$. На рис. 1 , представленном здесь, $\theta_{i}$ есть угол между продолжением прямой $X_{i-1} X_{i}$ за точку $X_{i}$ и лучом $X_{i} X_{i+1}$. Величина $\theta_{i}$ назьвается поворотом ломаной $L$ в ее вершине $X_{i}$. В точках $X_{0}$ и $X_{m}$ поворот ломаной считаем не определенным.

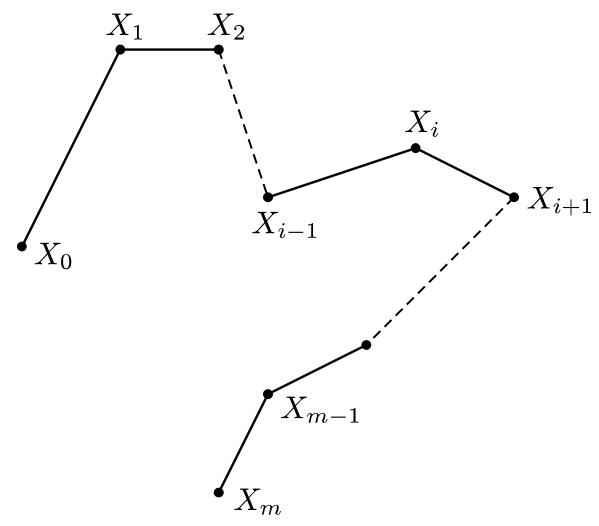

Рис. 1

Сумма

$$
\varkappa(L)=\sum_{i=1}^{m-1} \theta_{i}
$$

называется поворотом или интегральной кривизной ломаной.

Далее будет определено понятие поворота произвольной кривой в пространстве $\mathbb{R}^{n}$ и доказано, что для кривых, удовлетворяюших требованиям регулярности, принятым в дифференщиальной геометрии, интегральная кривизна действительно совпадает с интегралом от кривизны в смысле дифференциальной геометрии по длине дуги. Так что в теории А. Д. Александрова соблюден своего рода "принцип соответствия" - для регулярных кривых предложенные Александром Даниловичем построения приводят к тому же результату, что и построения классической дифференциальной геометрии.

Так как мы рассматриваем еще и замкнутые кривые, то полезно ввести понятие замкнутой ломаной. Ломаная в пространстве $\mathbb{R}^{n}$ с вершинами $X_{0}, X_{1}, \ldots, X_{m}$ - замкнутая, если $X_{0}=X_{m}$. Точки $X_{0}$ и $X_{m}$ считаются за одну и ту же вершину ломаной. В этом случае полагаем $\theta_{m}=\angle\left(\mathbf{a}_{m}, \mathbf{a}_{0}\right)$ и

$$
\varkappa(L)=\sum_{i=1}^{m} \theta_{i} .
$$

Пусть $L$ и $K-$ две произвольные ломаные. Тогда если ломаная $L$ вписана в ломаную $K$, то имеет место неравенство

$$
\varkappa(L) \leqslant \varkappa(K) .
$$

Поворот ломаной ведет себя в данном случае подобно длине - длина вписанной ломаной, как хорошо известно, не превосходит длины ломаной, в которую она вписана. 


\section{3. Поворот кривой, свойства кривых конечного поворота}

Пусть $K$ есть кривая в пространстве $\mathbb{R}^{n}$. Поворотом или интегральной кривизной кривой $K$ назьвается точная верхняя граница $\varkappa$ поворотов вписанных в нее ломаных. Допускается значение $\varkappa(K)=\infty$.

Интерес представляют в первую очередь те кривые, поворот которых конечен. Что можно сказать о таких кривых?

Пусть $K$ есть кривая в $\mathbb{R}^{n}, x:[a, b] \rightarrow \mathbb{R}^{n}$-произвольная ее параметризация. Пусть $t_{1}, t_{2} \in[a, b]$ таковы, что $x\left(t_{1}\right) \neq x\left(t_{2}\right)$. Проведем через точки $x\left(t_{1}\right)$ и $x\left(t_{2}\right)$ прямую и ориентируем ее следующим образом.

Если $t_{1}<t_{2}$, то считаем, что эта прямая имеет направление вектора $x\left(t_{2}\right)-x\left(t_{1}\right)$.

Если же $t_{2}<t_{1}$, то прямой $x\left(t_{1}\right) x\left(t_{2}\right)$ придаем направление вектора $x\left(t_{1}\right)-x\left(t_{2}\right)$.

Ориентированную таким образом прямую $x\left(t_{1}\right) x\left(t_{2}\right)$ мы будем далее обозначать символом $l\left(t_{1}, t_{2}\right)$ и назьвать секущей кривой $K$, проходящей через точки $x\left(t_{1}\right)$ и $x\left(t_{2}\right)$. Пусть $t_{0}<b$. Ориентированная прямая $\mathbf{l}_{r}$ назьвается правой касательной в сильном смысле в точке $x\left(t_{0}\right)$ кривой $K$, если угол $\angle\left[\mathbf{1}_{r}, l\left(t_{1}, t_{2}\right)\right]$ стремится к нулю, когда $t_{1} \rightarrow$ $t_{0}+0$ и $t_{2} \rightarrow t_{0}+0$. Аналогично определяется понятие левой касательной в сильном смысле в точке $x\left(t_{0}\right)$, где $A<t_{0}$, кривой $K$.

Кривая называется односторонне гладкой, если в каждой точке $x(t)$ при $a<t$ кривая имеет левую касательную в сильном смысле, а при $t<b$-правую касательную в сильном смысле.

Предположим, что в точке $x(t)$ кривой $K$ существуют левая и правая касательные в сильном смысле $\mathbf{l}_{r}(t)$ и $\mathbf{l}_{l}(t)$. Если эти касательные не совпадают, то говорят, что $x(t)$ есть угловая точка кривой $K$. Если $\mathbf{l}_{r}(t)=\mathbf{l}_{l}(t)$, то говорят, что $x(t)$ есть гладкая точка кривой $K$.

Введем еще обозначения. Символом $\mathbf{e}_{l}(X)$ обозначим единичный вектор, параллельньй и одинаково направленньй с левой касательной в точке $X=x(t)$ кривой $K$. Далее, пусть $\mathbf{e}_{r}(X)$ есть единичный вектор правой касательной в той же точке $X$ кривой $K$.

Справедливо следующее предложение.

ЛЕмма 1. Всякая односторонне гладкая кривая в пространстве $\mathbb{R}^{n}$ спрямляема и состоит из конечного числа простых дуг, и множество угловых точек односторонне гладкой кривой - не более чем счетно.

Доказательство элементарно, и я позволю себе на нем не останавливаться.

Далее $\mathbb{S}^{n-1}$ означает единичную сферу $\left\{x \in \mathbb{R}^{n}|| x \mid=1\right\}$ пространства $\mathbb{R}^{n}$. Для всякой односторонне гладкой кривой в пространстве $\mathbb{R}^{n}$ может быть определена некоторая кривая на сфере $\mathbb{S}^{n-1}$, называемая ее индикатрисой касательных.

Формальное построение индикатрисы касательных несколько громоздко. Я не буду его приводить, а ограничусь перечислением свойств индикатрисы касательных. Из этого перечисления нетрудно усмотреть, как эта самая индикатриса должна строиться.

Итак, пусть $K$ есть односторонне гладкая кривая в пространстве $\mathbb{R}^{n}$. Тогда определена кривая $T_{K}$ на сфере $\mathbb{S}^{n-1}$ такая, что вьполнены следующие условия. Каждой 
точке $X$ кривой $K$ может быть сопоставлено некоторое множество $\tau(X)$ точек кривой $T_{K}$ так, что выполнены следуюшие условия.

1) Если точка $X$ на кривой $K$ лежит левее точки $Y$, то каждая точка множества $\tau(X)$ лежит левее любой точки множества $\tau(Y)$.

$2)$ Если $X$ - угловая точка, то $\tau(X)$ есть кратчайшая дуга окружности большого круга, соединяющая точки $\mathbf{e}_{l}(X)$ и $\mathbf{e}_{r}(X)$ сферы $\mathbb{S}^{n-1}$. Если $X-$ гладкая точка кривой $K$, то $\tau(X)=\mathbf{e}(X)=\mathbf{e}_{l}(X)=\mathbf{e}_{r}(X)$.

$3)$ Всякая точка кривой $T_{K}$ принадлежит, по крайней мере, одной из дуг $\tau(X)$.

Если $K$ есть гладкая кривая, т.е. $K$ не имеет угловых точек и $x(s), 0 \leqslant s \leqslant S$, есть ее параметризация, где параметр $s$ есть длина дуги, то $x^{\prime}(s), 0 \leqslant s \leqslant S$, есть параметризаџия индикатрисы касательных.

ТЕОРема 1. Всякая кривая конечного поворота в пространстве $\mathbb{R}^{n}$ является односторонне гладкой, и ее поворот равен длине индикатрисы касательных кривой.

Теорема 1, как я говорил, принадлежит А.Д. Александрову. Из нее, во-первых, следует, что поворот регулярной кривой равен интегралу от кривизны по длине дуги. Действительно, если кривая $K$ удовлетворяет требованиям регулярности, принятым в дифференциальной геометрии, и $x(s), 0 \leqslant s \leqslant S$, есть ее параметризация, в которой параметр $s$ есть длина дуги, то $\left|x^{\prime \prime}(s)\right|=k(s)$, где $k(s)$ есть кривизна в точке $x(s)$ кривой $K$. Функция $y(s) \equiv x^{\prime}(s)$ есть параметризация индикатрисы касательных $T_{K}$ кривой $K$. Длина кривой $T_{K}$ равна

$$
\int_{0}^{S}\left|y^{\prime}(s)\right| d s=\int_{0}^{S} k(s) d s .
$$

В силу теоремы 1 , отсюда получаем, что в рассматриваемом случае поворот кривой равен интегралу от кривизны кривой по длине дуги. Для ломаной длина индикатрисы касательных, очевидно, равна повороту ломаной. Тем самым установлено, что наше определение интегральной кривизны ломаной согласуется с понятием интегральной кривизны кривой в классической дифференциальной геометрии.

Второе следствие теоремы 1 состоит в том, что поворот кривой может быть определен следующим образом. Пусть, для простоты, $K$ есть односторонне гладкая кривая. Будем рассматривать последовательности касательных $\mathbf{l}_{0}, \mathbf{l}_{1}, \ldots, \mathbf{l}_{m}$ кривой $K$ в точках, соответствуюших значениям параметра $t_{0}<t_{1}<\cdots<t_{m}$ кривой $K$. Тогда поворот кривой $K$ есть точная верхняя гранища сумм

$$
\sum_{i=1}^{m-1} \angle\left(\mathbf{l}_{i-1}, \mathbf{l}_{i}\right)
$$

Справедливость этого следует из того, что сумма, стоящая здесь, равна длине некоторой “сферической” ломаной (т.е. кривой, составленной из конечного числа кратчайших дуг окружности большого круга на сфере), вписанной в индикатрису касательных данной кривой. От требования, что кривая является односторонне гладкой, легко освободиться, понимая термин “касательная" в некотором обобщенном смысле. 
Я опускаю описание некоторых простых свойств кривых конечного поворота. Отмечу, что доказательство многих из них существенно облегчается благодаря тому, что вьполняется неравенство (2). Это обстоятельство верно также, если вместо евклидова пространства мы будем рассматривать пространство Н. И. Лобачевского.

Однако для кривых в пространстве постоянной положительной кривизны аналог неравенства (2), вообще говоря, не имеет места.

Отмечу некоторые из результатов относительно поворота кривой.

Следуюшая оценка получена Александром Даниловичем Александровым [1], [2] .

Tеорема 2. Пусть А и $B$ - две различные точки пространства $\mathbb{R}^{n}, l-$ расстояние между ними. Пусть дано число $\varkappa<\pi$. Тогда для всякой кривой $K$,

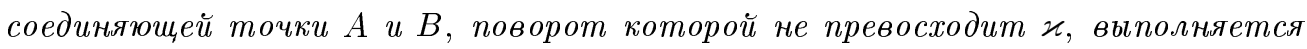
неравенство

$$
s(K) \leqslant \frac{l}{\cos \varkappa} .
$$

Равенство достигается в том и только том случае, когда кривая есть ломаная, образованная боковыми сторонами равнобедренного треугольника с основанием $А В$ и углом $\pi$ - и при его вершине.

В регулярном случае неравенство (3) было известно, по-видимому, еше в 30-е годы прошлого века. Существование кривой, для которой имеет место равенство, может быть доказано только на основе построений, предложенных А. Д. Александровым [1], [2].

Символом $D(K)$ будем обозначать диаметр кривой $K$. Пусть $\Delta(K)$ есть радиус наименьшего замкнутого шара в $\mathbb{R}^{n}$, содержашего кривую $K$.

Из оценки теоремы 2 вытекает оценка длины кривой через ее поворот и диаметр.

СлЕДСТВИЕ [3]. Для всякой кривой $K$ в пространстве $\mathbb{R}^{n}$ имеет место неравенство

$$
s(K) \leqslant \frac{D(K)}{\cos \alpha}\left\{\mathscr{E}\left(\frac{2 \varkappa(K)}{\alpha}\right)+1\right\} .
$$

Здесь $\alpha \in(0, \pi / 2)$ - произвольно, а $\mathscr{E}(x)$ означает челую часть числа $x$.

Оценка (4) не является точной. Если вместо $D(K)$ взять величину $\Delta(K)$, то оказывается возможньм получить точную оценку, как показывает следующая теорема.

Tеорема 3 (Ю.Г. Решетняк [6]). Для всякой кривой конечного поворота имеют место следующие неравенства:

$$
\begin{array}{llll}
s(K) \leqslant \frac{\Delta(K)}{\cos [\varkappa(K) / 2]} & \text { nрu } & \varkappa(K) \leqslant \frac{\pi}{2}, \\
s(K) \leqslant 2 \Delta(K) \sin [\varkappa(K) / 2] & \text { nрu } & \frac{\pi}{2} \leqslant \varkappa(K) \leqslant \frac{2 \pi}{3}
\end{array}
$$

и, наконеи,

$$
s(K) \leqslant \frac{\Delta(K)}{2}\left\{\varkappa(K)-\frac{2 \pi}{3}+2 \sqrt{3}\right\} \quad \text { nрu } \quad \varkappa(K)>\frac{2 \pi}{3} .
$$

Неравенства теоремь - точные. 
Теорема 4. Предположим, что последовательность кривых $K_{\nu}, \nu=1,2, \ldots$, сходится к некоторой кривой $K$ (т.е. надлежсащим образом выбранные параметризачии кривых $K_{\nu}$ сходятся равномерно к некоторой параметризачии кривой $K)$. Тогда имеет место неравенство

$$
\varkappa(K) \leqslant \underset{\nu \rightarrow \infty}{\lim } \varkappa\left(K_{\nu}\right) .
$$

Если последовательность поворотов кривых $K_{\nu}$ ограничена сверху, то длинь кривых $K_{\nu}$ имеют пределом длину кривой $K$.

ТЕОРема 5. Если кривая $K$ в пространстве $\mathbb{R}^{n}$ имеет конечный поворот, то и любая ее дуга является кривой конечного поворота. Пусть кривая $K$ точкой С разбивается на две дуги $K_{1}$ и $K_{2}$. Тогда если каждая из этих дуг является кривой конечного поворота, то $K$ есть кривая конечного поворота. При этом $\varkappa(K)=\varkappa\left(K_{1}\right)+\varkappa\left(K_{2}\right)+\theta$, где $\theta$ есть угол между левой и правой касательньми в точке $C$ кривой $K$.

Известна теорема Фенхеля, которая утверждает, что для всякой замкнутой кривой $K$ всегда $\varkappa(K) \geqslant 2 \pi$. В рамках данной теории она прямо следует из определения поворота. Пусть $X$ и $Y$ - две произвольные точки замкнутой кривой $K$, рассмотрим вписанную в нее замкнутую ломаную $L=X Y X$. Поворот этой ломаной равен $2 \pi$ и, значит, по определению, $\varkappa(K) \geqslant \varkappa(L)=2 \pi$.

\section{4. Основное интегрально-геометрическое соотношение для поворота кривой}

То, о чем я рассказал выше, - это все, что было сделано Александром Даниловичем, когда в 1949 г., будучи студентом 2-го курса математико-механического факультета Ленинградского университета, я приступил к работе над теорией кривых.

В июне 1952 г., готовясь к кандидатскому экзамену по философии, я в какой-то момент решил сделать небольшой перерьв и заняться математикой. Тут я обратил внимание на статью моего старшего коллеги по геометрическому семинару Виктора Абрамовича Залгаллера [5] (он был участником Великой Отечественной войны, участвовал в обороне Ленинграда, после войны активно занимался математикой). В этой работе было доказано некоторое интегрально-геометрическое соотношение относительно длины кривой. Я задался вопросом, а не верна ли аналогичная теорема для интегральной кривизны и интегрального кручения? Когда я написал аналог формул В. А. Залгаллера для поворота и интегральной кривизны, то мне сразу стало ясно, как преодолеть те трудности в теории кривых, которые я долго не мог осилить. Я понял, что полученные мною интегрально-геометрические соотношения позволяют сводить доказательства того или иного предложения к рассмотрению его частного случая, касающегося кривых простейшей природы. Быстро прикинув, как можно все это сделать, я рассказал о своих соображениях на ближайшем заседании геометрического семинара.

Пусть $K$ есть кривая в пространстве $\mathbb{R}^{n}$ и $\mathbf{n}$ есть произвольньй единичньй вектор в пространстве $\mathbb{R}^{n}$. Символом $K_{\mathbf{n}}$ обозначим ортогональную проекцию кривой на произвольную прямую, параллельную вектору $\mathbf{n}$. 
Опишем этот объект точнее. Пусть $x:[a, b] \rightarrow \mathbb{R}^{n}$ есть какая-либо параметризация кривой $K$. Тогда определена вектор-фрункция

$$
\langle\mathbf{n}, x(t)\rangle \mathbf{n}
$$

$a \leqslant t \leqslant b$. Она определяет в $\mathbb{R}^{n}$ некоторую кривую. От выбора параметризашии $x$ исходной кривой $K$ эта кривая не зависит. Обозначим ее символом $K_{\mathbf{n}}$ и назовем проекцией кривой $K$ на прямую, параллельную вектору $\mathbf{n}$ и проходящую через точку 0.

Возникает, таким образом, объект, в некотором роде парадоксальный, - кривая, лежащая в одной прямой и, вообше говоря, не являющаяся отрезком! Основная интегрально-геометрическая теорема о повороте кривой в рамках данной теории такова.

ТЕОРема 6. Пусть K есть произвольная кривая в пространстве $\mathbb{R}^{n}$. Тогда имеет место равенство

$$
\varkappa(K)=\frac{1}{\omega_{n}} \int_{\mathbb{S}^{n-1}} \varkappa\left(K_{\mathbf{n}}\right) d \omega(\mathbf{n}),
$$

где функция множества $\omega$ есть поверхностная мера $\left(\right.$ пощадь) на сфере $\mathbb{S}^{n-1}$, $\omega_{n}=\omega\left(\mathbb{S}^{n-1}\right)-$ полная площадь этой сферьи.

Конечность поворота кривой $K$ в теореме не предполагается.

Отметим, что если кривая лежит в одной прямой и имеет конечньй поворот, то она является ломаной из конечного числа звеньев и в каждой из ее вершин поворот равен $\pi$.

Доказательство теоремы основано на использовании следующего предложения.

Лемма 2. Пусть а $и \mathbf{b}-$ произвольные ненулевье векторы в $\mathbb{R}^{n} u \mathbf{a}_{\mathbf{n}} u \mathbf{b}_{\mathbf{n}}$ есть их ортогональные проекции на прямую, проходящую через точку 0 параллельно единичному вектору $\mathbf{n}$. Тогда имеет место равенство

$$
\angle(\mathbf{a}, \mathbf{b})=\frac{1}{\omega_{n}} \int_{\mathbb{S}^{n-1}} \angle\left(\mathbf{a}_{\mathbf{n}}, \mathbf{b}_{\mathbf{n}}\right) d \omega(\mathbf{n}) .
$$

Из леммы 2 вытекает справедливость теоремы 6 для ломаных. В общем случае теорема 6 вьводится отсюда предельным переходом с помошю теоремы Лебега о предельном переходе под знаком интеграла.

Несколько слов относительно истории вопроса. Теорема, аналогичная теореме 6 , была опубликована И. Фари в 1949 и 1951 гг. [26], [27]. И. Фари рассматривал кривые в $\mathbb{R}^{3}$ и под $K_{\mathbf{n}}$ понимал ортогональную проекцию кривой на плоскость с нормалью n. Для регулярных кривых теорема 6 была доказана Дж. Милнором в 1950г. [28]. Дж. Милнор не располагал понятием поворота кривой, лежащей в одной прямой. Под $\varkappa\left(K_{\mathbf{n}}\right)$ он понимал умноженное на $\pi$ число внутренних точек экстремума функции $\langle x(t), \mathbf{n}\rangle$, где $x(t)$ - произвольная параметризация кривой $K$. И. Фари и Дж. Милнором были указаны некоторые топологические приложения теоремы 6.

Я пришел к теореме 6 независимо от И. Фари и Дж. Милнора в 1952 г. Мои результаты были опубликованы только в 1957 г. [8] (часть из них вошла в мою кандидатскую диссертацию 1954 г.). 
Теорема 6 была применена мною к задачам, в основном отличающимся от тех, которые изучались И. Фари и Дж. Милнором. Укажем некоторые из таких задач.

Пусть $K$ есть кривая конечного поворота, $x:[a, b] \rightarrow \mathbb{R}^{n}-$ произвольная параметризация этой кривой.

Двойной цепочкой на отрезке $[a, b]$ назьвается всякая конечная последовательность $\zeta$ пар точек $\left\{t_{i}, u_{i}\right\}, i=1, \ldots, m$, такая, что при каждом $i$ вьполнено неравенство $t_{i}<u_{i}$ и $a \leqslant t_{1} \leqslant t_{2} \leqslant t_{m} \leqslant b$ и $a \leqslant u_{1} \leqslant u_{2} \leqslant u_{m} \leqslant b$. Кратностью двойной цепочки назовем наибольшее из чисел $N$ таких, что сушествует значение $t \in[a, b]$, принадлежашее, по крайней мере, $N$ отрезкам $\left[t_{i}, u_{i}\right]$.

Наконец, введем еще величину, характеризующую одновременно густоту расположения точек $t_{i}$ и $u_{i}$ на отрезке $[a, b]$ и близость точек $t_{i}$ и $u_{i}$. А именно, полагаем

$$
\delta(\zeta)=\max \left\{u_{1}-a, u_{2}-t_{1}, \ldots, u_{i+1}-t_{i}, b-t_{m}\right\}
$$

Предположим, что задана кривая $x:[a, b] \rightarrow \mathbb{R}^{n}$, и пусть $\zeta-$ двойная цепочка на промежутке $[a, b]$. Тогда определена конечная последовательность секущих $\Lambda(\zeta, x)=$ $\left\{\mathbf{l}\left(t_{i}, u_{i}\right), i=1, \ldots, m\right\}$. Положим

$$
\varkappa[\Lambda(\zeta, x)]=\sum_{i=1}^{m-1} \angle\left(\mathbf{l}\left(t_{i}, u_{i}\right), \mathbf{l}\left(t_{i+1}, u_{i+1}\right)\right) .
$$

Теорема 7 (Ю.Г. Решетняк). Пусть $x:[a, b] \rightarrow \mathbb{R}^{n}$ есть кривая конечного поворота в пространстве $\mathbb{R}^{n} u\left(\zeta_{\nu}\right)_{\nu \in \mathbb{N}}-$ последовательность двойных чепочек промежутка $[a, b]$. Предположим, что $\delta\left(\zeta_{\nu}\right) \rightarrow 0$ при $\nu \rightarrow \infty$ и существует постоянная $M<\infty$ такая, что кратность каждой из двойных цепочек $\zeta_{\nu}$ не превосходит $M$. Тогда суммы $\left[\Lambda\left(\zeta_{\nu}, x\right)\right]$ имеют своим пределом поворот данной кривой.

Теорема 7 содержит решение проблемы об аппроксимации поворота суммами углов между последовательньми секущими, поставленной А. Д. Александровым. Доказательство основано на следуюших соображениях. Сначала устанавливается, что теорема верна для кривых конечного поворота, лежаших в одной прямой. Каждая такая кривая есть ломаная, состояшая из конечного числа звеньев, и в каждой ее вершине поворот равен $\pi$. В этом случае требуемьй результат доказьвается элементарно. При этом ограничения, касаюшиеся кратности, являются излишними. В результате получаем, что для всякого вектора $\mathbf{n}$, для которого $\varkappa\left(K_{\mathbf{n}}\right)<\infty$, имеет место равенстBо

$$
\varkappa\left(K_{\mathbf{n}}\right)=\lim _{\nu \rightarrow \infty} \varkappa\left[\Lambda\left(\xi_{\nu},\left(x_{\nu}\right)_{\mathbf{n}}\right)\right] .
$$

Положим $\Phi_{\nu}(\mathbf{n})=\varkappa\left[\Lambda\left(\xi_{\nu},\left(x_{\nu}\right)_{\mathbf{n}}\right)\right]$. Применяя равенство $(1)$, получим, что

$$
\frac{1}{\omega_{n}} \int_{\mathbb{S} n-1} \Phi_{\nu}(\mathbf{n}) d \omega(\mathbf{n})=\varkappa\left[\Lambda\left(\xi_{\nu}, x\right)\right] .
$$

При $\nu \rightarrow \infty$ для всякого $\mathbf{n}$, для которого $\varkappa\left(K_{\mathbf{n}}\right)$ конечно, величина $\Phi_{\nu}(\mathbf{n}) \rightarrow \varkappa\left(K_{\mathbf{n}}\right)$. Осталось заметить, что имеет место неравенство

$$
\Phi_{\nu}(\mathbf{n}) \leqslant 2(M+1)\left[\varkappa\left(K_{\mathbf{n}}\right)+\pi\right]
$$


где $M<\infty$ - постоянная. В силу конечности поворота кривой $K$, правая часть этого неравенства интегрируема по сфере $\mathbb{S}^{n-1}$. Ограничение на кратности двойных цепочек гарантирует нам, таким образом, выполнение условий теоремы Лебега о предельном переходе под знаком интеграла.

Теорема 6 позволяет доказать также некоторое утверждение о строении "типичных" точек плоской кривой.

Пусть $K$ есть кривая на плоскости. Точку $X$ кривой $K$ назовем точкой локальной вьпуклости, если найдутся окрестность $U$ точки $X$ и прямая $l$, проходящая через точку $X$, такие, что $U \cap K$ есть простая дуга, лежашая по одну сторону $l$, и всякая прямая, параллельная $l$, пересекает дугу $U \cap K$ не более чем в двух точках, причем если этих точек две, то они лежат по разные стороны точки $X$. Рис. 2 иллюстрирует данное определение.

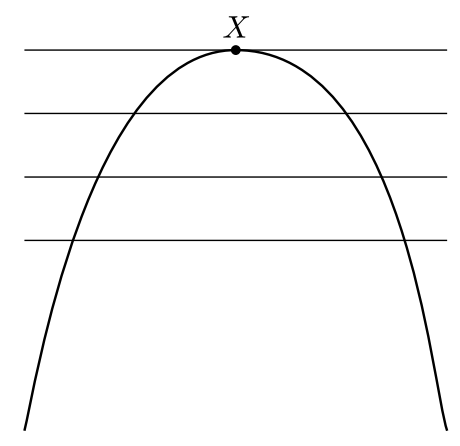

Рис. 2

Если $K$ есть кривая конечного поворота, то на ней может быть определена некоторая мера $\varkappa$ такая, что для всякой открытой дуги $(X Y)$ данной кривой величина $\varkappa[(X Y)]$ равна повороту дуги $[X Y]$ как самостоятельной кривой в $\mathbb{R}^{2}$. Если множество $E$ точек кривой $K$ состоит из единственной точки $X$, то $\varkappa(E)$ равно углу $\theta(X)$ между левой и правой касательньми в точке $X$ кривой $K$.

Теорема 8. Пусть К есть плоская кривая конечного поворота. Тогда почти

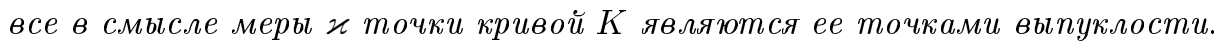

Применяя теорему 6 и теорему работы В.А. Залгаллера [5], можно получить следуюшую оценку длины кривой через ее поворот и диаметр:

$$
s(K) \leqslant C_{n} d(K)[\varkappa(K)+\pi]
$$

где $d(K)$ - диаметр кривой, а $C_{n}=\frac{\Gamma\left[\frac{n+1}{2}\right]}{\sqrt{\pi} \Gamma\left[\frac{n}{2}\right]}-$ постоянная.

Утверждение теоремы 4 о сходимости длин допускает некоторое количественное уточнение. Пусть $K_{1}$ и $K_{2}$ - две кривые в пространстве $\mathbb{R}^{n}$. Выберем произвольно параметризацию $x:[a, b] \rightarrow \mathbb{R}^{n}$ кривой $K_{1}$ и параметризацию $Y:[c, d] \rightarrow \mathbb{R}^{n}$ кривой $K_{2}$. Пусть $\varphi:[a, b] \rightarrow \mathbb{R}$ есть непрерьвная строго возрастаюшая функция такая, что $\varphi(a)=c$, а $\varphi(b)=d$. Пусть $\rho_{\varphi}(x, y)=\sup _{t \in[a, b]}|x(t)-y[\varphi(t)]|$. Точная нижняя 
гранища величины $\rho_{\varphi}(x, y)$ на множестве всех $\varphi$, удовлетворяюших указанньм вьше условиям, не зависит от выбора параметризаций $x$ и $y$ данных кривых, назьвается расстоянием между кривыми $K_{1}$ и $K_{2}$ и обозначается символом $\rho\left(K_{1}, K_{2}\right)$. Легко проверяется, что $\rho$ есть метрика на множестве всех кривых пространства $\mathbb{R}^{n}$. Сходимость последовательности кривых в смысле теоремы 4 есть сходимость относительно построенной метрики $\rho$. Справедливо следуюшее неравенство:

$$
\left|s\left(K_{1}\right)-s\left(K_{2}\right)\right| \leqslant 2 C_{n} \rho\left(K_{1}, K_{2}\right)\left[\varkappa\left(K_{1}\right)+\varkappa\left(K_{2}\right)+\pi\right]
$$

где $C_{n}$ есть та же постоянная, что и в неравенстве (7). Действительно, пусть кривые $K_{\nu}$ сходятся к кривой $K$ и их повороты не превосходят постоянной $M<\infty$. Тогда, в силу первого утверждения теоремы 4 , поворот кривой $K$ также не превосходит $M$. Отсюда заключаем, что

$$
\left|s\left(K_{\nu}\right)-s(K)\right| \leqslant 2 C_{n} \rho\left(K_{\nu}, K\right)[2 M+\pi]
$$

Так как $\rho\left(K_{\nu}, K\right) \rightarrow 0$ при $\nu \rightarrow \infty$, мы получаем

$$
s(K)=\lim _{\nu \rightarrow \infty} s(K)
$$

что и требовалось доказать.

Понятие кривой конечного поворота в математике впервые появилось в работе И. Радона [29], посвященной теории потенциала на плоскости. Он рассматривал области на плоскости, граница которых есть (в нашей терминологии) кривая конечного поворота. Известна также теорема И.М. Либермана [23], ученика и сотрудника А. Д. Александрова, погибшего на фронте в Великой Отечественной войне. Согласно теореме И.М. Либермана, геодезическая на произвольной выпуклой поверхности есть, как мы говорим сейчас, кривая конечного поворота. Свойства кривых конечного поворота существенно использовались знаменитьм учеником А. Д. Александрова Алексеем Васильевичем Погореловым в его исследованиях (см. [24]) по изгибанию вьпуклых поверхностей (Алексей Васильевич Погорелов называл их кривыми с ограниченной вариацией поворота).

В работе А.Г. Хованского и С. Ю. Яковенко [30] для кривых в $\mathbb{R}^{n}$ устанавливается некоторое неравенство. Доказательство этих авторов использует приведенную выше теорему 6 (в редакции Дж. Милнора). Определение интегральной кривизны по А.Д. Александрову позволяет получить теорему А.Г. Хованского и С. Ю. Яковенко средствами элементарной геометрии, см. [25].

\section{5. Интегральное кручение кривой}

Сначала сделаем некоторые замечания относительно того, как определять угол между плоскостями в $\mathbb{R}^{3}$.

Пусть $P$ и $Q$ - две ориентированные плоскости в $\mathbb{R}^{3}$. Если эти плоскости, как подмножества $\mathbb{R}^{3}$, совпадают, то угол между ними считаем равншм нулю в случае, если их ориентации также совпадают, и равньм $\pi$, если их ориентации противоположны. 
Предположим, что плоскости $P$ и $Q$ не параллельны и пересекаются по некоторой прямой $l$. Допустим, что задана некоторая ориентация $l$. Пусть $\alpha(P, Q)>0$ есть наименьший угол, на которьй надо повернуть плоскость $P$, чтобы совместить ее с плоскостью $Q$ кратчайшим путем. Пусть $\sigma(P, Q)=1$, если указанное направление вращения вместе с направлением прямой $l$ образует правый винт, и $\sigma(P, Q)=-1$, если этот винт левьй. Полагаем $\angle(P, Q)=\sigma(P, Q) \alpha(P, Q)$.

При $n>3$ понятие правого или левого винта не имеет смысла. Если $P$ и $Q-$ две ориентированные плоскости в пространстве $\mathbb{R}^{n}$, где $n \geqslant 4$, пересекающиеся по одной прямой, то эти плоскости содержатся в некоторой трехмерной плоскости, и в этом случае считаем, что угол $\angle(P, Q)$ между плоскостями $P$ и $Q$ всегда положителен и равен величине, обозначенной вьше символом $\alpha(P, Q)$.

Пусть дана ломаная $L$ в пространстве $\mathbb{R}^{n}$, а $X_{i}, i=0,1, \ldots, m,-$ ее последовательные вершины. Предположим, что точки $X_{i-1}, X_{i}$ и $X_{i+1}$ не лежат в одной прямой. Пусть $P_{i}$ есть двумерная плоскость, проходящая через эти точки и ориентированная так, чтобы пара векторов $\left(\mathbf{a}_{i}, \mathbf{a}_{i+1}\right)$ была в ней правой парой. Ориентированная так плоскость $P_{i}$ назьвается соприкасающейся плоскостью в точке $X_{i}$ ломаной $L$. (Напомню, что $\mathbf{a}_{i}=\overrightarrow{X_{i-1} X_{i}}$, a $\mathbf{a}_{i+1}=\overrightarrow{X_{i} X_{i+1}}$.)

Может оказаться, что для некоторых $i$ точки $X_{i-1}, X_{i}$ и $X_{i+1}$ лежат в одной прямой. Пусть $i_{1}<i_{2}<\cdots<i_{k}-$ все те значения $i$, для которых точки $X_{i-1}, X_{i}$ и $X_{i+1}$ не лежат в одной прямой. Если таких значений $i$ нет, то данная ломаная лежит в одной прямой и понятие интегрального кручения для нее не определено.

Будем считать, что данная ломаная не лежит в одной прямой. Тогда определены плоскости

$$
P_{i_{0}}, P_{i_{1}}, \ldots, P_{i_{k}} \text {. }
$$

Две соседние плоскости $P_{i_{j}}$ и $P_{i_{j+1}}$ в последовательности (8) пересекаются по некоторой прямой.

Далее следует различать случаи $n=3$ и $n>3$. В случае $n=3$ эту прямую необходимо ориентировать. Выбор ориентации есть вопрос соглашения. Векторы $\mathbf{a}_{i_{j}+1}$ и $\mathbf{a}_{i_{j+1}}$ лежат в прямой, по которой пересекаются плоскости $P_{i_{j}}$ и $P_{i_{j+1}}$. Ориентируем прямую $P_{i_{j}} \cap P_{i_{j+1}}$ так, чтобы она была направлена одинаково с вектором $\mathbf{a}_{i_{j}+1}$.

Величина

$$
\tau(L)=\sum_{j=1}^{k} \angle\left(P_{i_{j}}, P_{i_{j+1}}\right)
$$

называется полным кручением ломаной $L$. Полагаем также

$$
|\tau|(L)=\sum_{j=1}^{k}\left|\angle\left(P_{i_{j}}, P_{i_{j+1}}\right)\right| .
$$

Величина $|\tau|(L)$ называется абсолютньм полным кручением ломаной $L$.

В случае ломаных в $\mathbb{R}^{n}$, где $n>3$, угол между плоскостями $P_{i_{j}}$ и $P_{i_{j+1}}$ всегда положителен. В этом случае в сумме (9) все слагаемые положительны и эта сумма называется также полным кручением ломаной. (Вероятно, в этом случае было бы более правильно назьвать сумму (9) второй интегральной кривизной ломаной $L$.) 
Пусть $K$ есть кривая в $\mathbb{R}^{n}$, не лежашая в одной прямой пространства $\mathbb{R}^{n}$. Тогда абсолютным полным кручением кривой $K$ назьвается верхний предел величин $|\tau|(L)$ для ломаных, вписанных в кривую $K$ и сходящихся к ней. Этот верхний предел обозначается символом $|\tau|(K)$.

Конечность абсолютного полного кручения обеспечивает наличие у кривой разного рода "хороших" свойств. Пусть $|\tau|(K)<\infty$. Тогда, если никакая правая полуокрестность точки $X$ кривой $K$ не лежит в одной прямой, то кривая имеет в этой точке соприкасающуюся плоскость в некотором сильном смысле (подробности читатель сможет найти в монографии [4]). Если всякая прямолинейная дуга кривой $K$ имеет поворот, равньй нулю, то поворот кривой $K$ конечен.

Исследование величины $|\tau|(K)$ встретилось с определенными трудностями. В частности, надо было доказать, что на самом деле $|\tau|(K)$ есть не верхний, а самьй “обычный” предел величины $|\tau|(L)$ для ломаных $L$, вписанных в кривую $K$ и сходяшихся к ней. В действительности, это утверждение верно лишш при некоторых дополнительных предположениях относительно кривой (см. [4]). Я смог найти доказательство этого только тогда, когда сообразил, что для кручения также имеет место интегрально-геометрическая теорема, аналогичная теореме 6 вьше. А именно, справедлива следуюшая теорема.

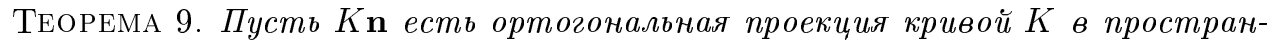
стве $\mathbb{R}^{3}$ на плоскость, проходящую через точку 0 и ортогональную единичному вектору п. Тогда имеет место равенство

$$
|\tau|(K)=\frac{1}{4 \pi} \int_{\mathbb{S}^{2}} \tau(K \mathbf{n}) d \omega(\mathbf{n})
$$

Теорема 9 (см. [4]) оказалась ключом ко всем тайнам интегрального кручения кривой! С ее помошю рассмотрение кривых в обшем случае сводится к простейшему случаю плоских кривых с конечным полным кручением.

В качестве комментария к теореме 9 стоило бы пояснить, что́ есть плоская кривая конечного полного кручения. В классической дифференциальной геометрии для плоской кривой кручение равно нулю. В нашем случае возникает некоторый парадоксальный объект - плоские кривые конечного и, вообще говоря, ненулевого полного кручения.

Приведем здесь описание таких кривых. Всякая плоская кривая конечного полного кручения устроена следуюшим образом - она состоит из конечного числа вьпуклых дуг, как, например, кривая, представленная на рис. 3.
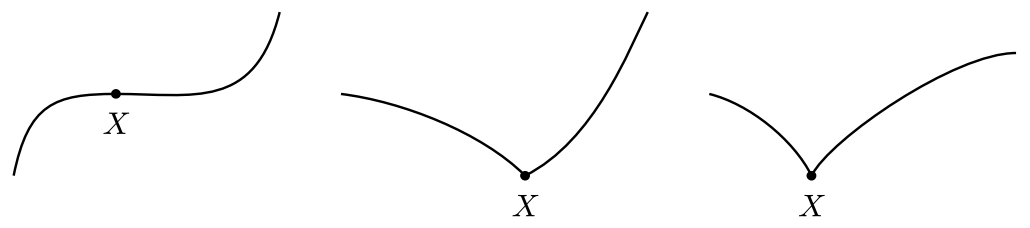

Рис. 3 
При переходе через точку, соединяющую две такие дуги, кручение меняется скачком на величину, равную одному из чисел $0, \pi$ или $2 \pi$. В случае, если вьпуклые дуги, соединяющиеся в точке $X$ кривой таковы, что дуга, лежашая слева от $X$, обращена вьпуклостью в одну сторону, а дуга справа от $X$ обращена вьпуклостью в сторону противоположную, то скачок полного кручения при переходе через точку $X$ равен $\pi$. Если же обе эти дуги направлены вьпуклостью в одну сторону, но никакая окрестность точки $X$ на кривой не является вьпуклой дугой, то скачок кручения при переходе через точку равен $2 \pi$. Если дуги кривой, примыкающие одна к другой в точке $X$ кривой, направлены вьпуклостью в одну сторону и некоторая окрестность точки $X$ вьпукла, то скачок кручения при переходе через точку $X$ равен 0 . Все возможные типы особенностей представлены на рис. 4.

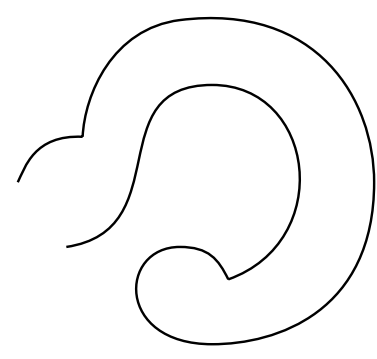

Рис. 4

Замечу, что возможное наличие в составе кривой прямолинейных отрезков несколько усложняет картину, но я не буду останавливаться на связанных с этим подробностях.

\section{6. Восстановление кривой по интегральной кривизне и интегральному кручению}

Некоторьй аналог формул Френе и теорема об однозначном с точностью до движения восстановлении кривой по ее повороту и кручению (для кривых в $\mathbb{R}^{3}$ ) имеют место и в рассматриваемой здесь теории кривых. При этом формулы Френе должны быть представлены в интегральной форме. Мы не приводим точные формулировки соответствующих утверждений, ввиду необходимости достаточно пространного описания всякого рода вспомогательных результатов.

\section{7. О теории нерегулярных кривых в $\mathbb{R}^{n}$}

Несколько слов о распространении теории кривых по А. Д. Александрову на случай кривых в пространстве $\mathbb{R}^{n}$ для произвольного $n$. Путь, изначально предложенньй Александром Даниловичем, ведет в этом случае к построениям, громоздкость которых с ростом размерности пространства растет.

Теория кривых по А.Д. Александрову имеет дело с множеством кривых, удовлетворяюших условиям ограниченности некоторых функционалов кривой.

Можно попытаться определить этот класс способом, отличным от предложенного А. Д. Александровым. Тогда подход А. Д. Александрова к теории кривых приведет 
к теоремам об аппроксимации функционалов кривых функщионалами вписанных ломаных. Все сложности теории кривых по А.Д. Александрову в этом случае будут перенесены в доказательства этих теорем об аппроксимации.

Способы построения теории кривых в многомерном случае рассматривались моим учеником, вьпускником Новосибирского университета Иваном Филипповичем Майником, [10]-[17], [20], [21].

В качестве альтернативного способа построения теории кривых в $n$-мерном евклидовом пространстве мною предложен подход к построению теории кривых, основанньй на понятиях индикатрисы касательных и развертки сферической кривой. Этому посвящена работа [22], опубликованная в 2001 г. Понятие индикатрисы касательных определено выше.

Пусть $K$ есть кривая в сферическом пространстве $\mathbb{S}^{n}$. Пространство $\mathbb{R}^{n}$ представим как плоскость в $\mathbb{R}^{n+1}$. Развертка кривой $K$ есть след, зачерчиваемый точкой касания сферы $\mathbb{S}^{n}$ в пространстве $\mathbb{R}^{n}$, ког да сфера катится без скольжения и проворачивания по плоскости $\mathbb{R}^{n}$, касаясь ее в точках кривой $K$. Сказанное есть лишш попытка наглядного описания понятия развертки. Точное определение опирается на понятие параллельного переноса вектора в римановом пространстве, см. [9].

В [22] для любых целых $n$ и $m$ таких, что $n \geqslant 1$ и $0 \leqslant m \leqslant n$, определены классы кривых $\mathscr{K}_{m}\left(\mathbb{R}^{n}\right)$ в пространстве $\mathbb{R}^{n}$ и классы $\mathscr{K}_{m}\left(\mathbb{S}^{n}\right)$ в $n$-мерном сферическом пространстве $\mathbb{S}^{n}$. При этом мы предполагаем, что при каждом $m$ для всякой кривой из $\mathscr{K}_{m}\left(\mathbb{R}^{n}\right)$ определено неотрицательное число $\varkappa_{m}(K)<\infty$ и для любой кривой $K \in \mathscr{K}_{m}\left(\mathbb{S}^{n}\right)$ определена конечная величина $\varkappa_{m}^{g}(K) \geqslant 0$. В случае $m=n-1$ для кривых классов $\mathscr{K}_{m}\left(\mathbb{R}^{n}\right)$ и $\mathscr{K}_{m}\left(\mathbb{S}^{n}\right)$ определены еще числа $(\varkappa)_{n-1}(K)$ и $(\varkappa)_{n-1}^{g}(K)$.

При каждом $n$ класс $\mathscr{K}_{0}\left(\mathbb{R}^{n}\right)$ есть множество всех спрямляемых кривых пространства $\mathbb{R}^{n}$ и, аналогично, $\mathscr{K}_{0}\left(\mathbb{S}^{n}\right)$ есть множество всех спрямляемых кривых на сфере $\mathbb{S}^{n}$. В этом случае $\varkappa_{0}(K)$ и $\varkappa_{0}^{g}(K)$ есть просто длина кривой $K$.

В случае $n=1$ и $m=0$ множество $\mathbb{S}^{1}$ есть окружность и в этом случае для кривой $K$ с параметризацией $z(t), a \leqslant t \leqslant b$, в $\mathbb{S}^{1}$ величина $(\varkappa)_{0}(K)$ равна приращению полярного угла точки $z(t)$, когда $t$ меняется от $a$ до $b$,

$$
(\varkappa)_{0}(K)=\left.\arg z(t)\right|_{a} ^{b}
$$

Предположим, что для некоторого целого $m \geqslant 0$ классы $\mathscr{K}_{m}\left(\mathbb{R}^{n}\right)$ и $\mathscr{K}_{m}\left(\mathbb{S}^{n}\right)$ определены для всякого $n \geqslant m$. Тогда мы будем говорить, что кривая $K$ в пространстве $\mathbb{R}^{n+1}$ принадлежит классу $\mathscr{K}_{m+1}\left(\mathbb{R}^{n}\right)$, если $K$ есть односторонне гладкая кривая и ее сферическая индикатриса касательных $K^{\prime}$ принадлежит классу $\mathscr{K}_{m}\left(\mathbb{S}^{n-1}\right)$. В этом случае полагаем $\varkappa_{m+1}(K)=\varkappa_{m}\left(K^{\prime}\right)$. В случае $m=n-2$ определена также величина $(\varkappa)_{m+1}(K)=(\varkappa)_{m}^{g}\left(K^{\prime}\right)$.

Кривая $K$ на сфере $\mathbb{S}^{n}$ принадлежит классу $\mathscr{K}_{m+1}\left(\mathbb{S}^{n}\right)$, если ее развертка $\widetilde{K}$ в $\mathbb{R}^{n}$ есть кривая класса $\mathscr{K}_{m+1}\left(\mathbb{R}^{n}\right)$. При этом мы полагаем $\varkappa_{m+1}^{g}(K)=\varkappa_{m+1}(\widetilde{K})$, а в случае $m=n-2$ определим еще величину $(\varkappa)_{m+1}^{g}(K)=(\varkappa)_{m+1}(\widetilde{K})$.

Функционалы $\varkappa_{m}$ и $\varkappa_{m}^{g}$ суть интегральные кривизны порядка $m$ в $\mathbb{R}^{n}$ и в $\mathbb{S}^{n}$ соответственно.

Для всякого $m>0$ и любого $n \geqslant m$ справедливы включения

$$
\mathscr{K}_{m}\left(\mathbb{R}^{n}\right) \subset \mathscr{K}_{m-1}\left(\mathbb{R}^{n}\right), \quad \mathscr{K}_{m}\left(\mathbb{S}^{n}\right) \subset \mathscr{K}_{m-1}\left(\mathbb{S}^{n}\right) .
$$


Всякая ломаная в $\mathbb{R}^{n}$, не лежашая в одной $(m-1)$-мерной плоскости, принадлежит классу $\mathscr{K}_{m}\left(\mathbb{R}^{n}\right)$. Если кривая $K$ допускает параметризацию $x:[a, b] \rightarrow \mathbb{R}^{n}$ такую, что вектор-функция $x$ принадлежит классу $\mathscr{C}^{m}([a, b])$, причем производная $x^{\prime}(t) \neq 0$ нигде не обрашается в нуль, то $K \in \mathscr{K}_{m}\left(\mathbb{R}^{n}\right)$. При этом $\varkappa_{m}(K)$ равно интегралу от $m$-й кривизны кривой по длине дуги.

Исследование свойств введенных здесь классов кривых $\mathscr{K}_{m}\left(\mathbb{R}^{n}\right)$ и, в частности, доказательство аналогов теорем 6 и 9 для величин $\varkappa_{m}(K)$, так же как и теорем об аппроксимации функционалов $\varkappa_{m}(K)$ значениями функционалов для ломаных, есть дело будушего.

\section{СПИСОК ЛИТЕРАТУРЫ}

[1] А. Д. Александров. Теория кривых на основе приближения ломаными // Научная сессия ЛГУ. Тезисы докладов. Секция матем. наук. Л.: ЛГУ, 1946.

[2] А. Д. Александров. Теория кривых на основе приближения ломаными // УМН. 1947. T. 2. № 3. C. $182-184$.

[3] А.Я. Юсупов. Теория кривых на основе приближения ломаными // Дисс.... канд. физ.-матем. наук. Л.: Пед. ин-т им. А. И. Герцена, 1949.

[4] I. Fáry. Sur la courbure totale d'une courbe gauche faisant un nœud // Bull. Soc. Math. France. 1949. V. 77. P. 128-138.

[5] J. Milnor. On the total curvature of knots // Ann. of Math. (2). 1950. V. 52. P. 248-257.

[6] I. Fáry. Sur certaines inégalités géométriques // Acta. Sci. Math. Szeged. 1950. V. 12. Part A. P. 117-124.

[7] В.А. Залгаллер. Вариации кривых вдоль фиксированных направлений // Изв. АН CССР. Сер. матем. 1951. Т. 15. № 5. С. 463-476.

[8] Ю. Г. Решетняк. О длине и повороте кривой и о площади поверхности // Дисс.... канд. физ.-матем. наук. Л.: ЛГУ, 1954.

[9] Ю.Г. Решетняк. Интегрально-геометрический метод в теории кривых // Труды 3-го Всесоюзного математического съезда (Москва, 1956). Т. 1: Секц. докл. М.: Изд-во АН CCCP, 1956. C. 164.

[10] Метод ортогональных проекций в теории кривых // Вестн. Ленингр. ун-та. Сер. матем., мех, астрон. 1957. № 13. Вып. 3. С. 22-26.

[11] Ю. Г. Решетняк. О понятии подъема нерегулярного пути в расслоенном многообразии и его приложениях // Сиб. матем. журн. 1975. Т. 16. № 3. С. 588-598.

[12] И.Ф. Майник. О монотонных кривых и интегральной геометрии в $n$-мерном евклидовом и сферическом пространствах // Деп. в ВИНИТИ, № 2194-76. М., 1976.

[13] И.Ф. Майник. О нерегулярных кривых в $n$-мерном евклидовом и сферическом пространствах // Деп. в ВИНИТИ, № 3184-76. М., 1976.

[14] И.Ф. Майник. О кривых в редуктивных пространствах // Докл. АН СССР. 1977. Т. 235. № 3. С. 531-533.

[15] И.Ф. Майник. Об $r$-вписанных кривых // Сиб. матем. журн. 1979. Т. 20. № 1. С. 95-108.

[16] И.Ф. Майник. Об интегральных кривизнах в $\mathbb{R}^{n}$ и $S^{n} / /$ Симпозиум по геометрии в целом и основам теории относительности: Тезисы докладов. Новосибирск: Наука, 1982. C. $53-54$.

[17] И.Ф. Майник. Проекционный признак равенства кривых // Сиб. матем. журн. 1983. T. 24. № 4. C. 100-103.

[18] И.Ф. Майник. Проекционньй признак равенства пространственных кривых // Сиб. матем. журн. 1985. Т. 26. № 2. С. 207-209.

[19] И.Ф. Майник. Об аппроксимации интегральных кривизн кривых в $R^{n}$ и $S^{n} / /$ Сиб. матем. журн. 1987. Т. 28. № 5. C. 128-133.

[20] А. Д. Александров, Ю. Г. Решетняк. Поворот кривой в $n$-мерном евклидовом пространстве // Сиб. матем. журн. 1988. Т. 29. № 1. С. 3-22. 
[21] Ю. Г. Решетняк. Некоторые применения интегральной геометрии к теории кривых конечного поворота // Сиб. матем. журн. 1988. Т. 29. №1. С. 141-150.

[22] A. D. Alexandrov, Yu. G. Reshetnyak. General Theory of Irregular Curves. Dordrecht: Kluwer, 1989.

[23] И.Ф. Майник. К оценке длины кривой спуска // Сиб. матем. журн. 1992. Т. 33. № 4. C. $215-218$.

[24] И.Ф. Майник. К устойчивости в теореме об оценке длины кривой спуска // Сиб. матем. журн. 1997. Т. 38. № 4. С. 867-875.

[25] Ю. Г. Решетняк. О теории нерегулярных кривых в $n$-мерном евклидовом пространстве // Геометрия и приложения: Труды конференции, посвященной 70-летию В. А. Топоногова (Новосибирск, 2000). Новосибирск: Изд-во ИМ СО РАН, 2001. С. 158-170.

[26] J. Radon. Über Randwertaufgaben beim logarithmischen Potential // Sitzber. Akad. Wiss. Wien. 1919. V. 128. P. 1123-1167.

[27] И. М. Либерман. Геодезические линии на выпуклых поверхностях // Докл. АН СССР. 1941. T. 32. № 5. С. 310-313.

[28] А. В. Погорелов. Внешняя геометрия выпуклых поверхностей. М.: Наука, 1969.

[29] A. G. Khovanskii, S. Yu. Yakovenko. Generalized Rolle theorem in $\mathbb{R}^{n}$ and $\mathbb{C} / / \mathrm{J}$. Dynam. Control Systems. 1996. V. 2. №1. P. 103-123.

[30] Ю. Г. Решетняк. Оценка угла обзора кривой в метрическом пространстве неположительной кривизны // Сиб. матем. журн. 2002. Т. 43. № 3. С. 694-701.

Институт математики им. С. Л. Соболева СО РАН

Поступила в редакцию 15.06 .2005 